IP Periodica Polytechnica Civil Engineering

59(3), pp. 337 346 2015

DOI: $10.3311 /$ PPci.7474

Creative Commons Attribution (1)

RESEARCH ARTICLE

\section{Numerical Study on the Flow over a Simplified Vehicle Door Gap - an Old Benchmark Problem Is Revisited}

\author{
Bence Farkas, György Paál
}

Received 08-04-2014, revised 18-09-2014, accepted 17-02-2015

\begin{abstract}
A simplified automobile door gap model, defined at the Third Computational Aeroacoustics (CAA) Workshop on Benchmark Problems (Category 6) was investigated. After a thorough mesh study compressible and incompressible simulations were carried out and various turbulence models were tried. The influence of three dimensional effects and boundary layer thickness effects were examined too. In case of compressible simulations stability problems were encountered with the non-reflective boundary condition of CFX (beta version at the time of the simulations). It was found that for deep cavities incompressible simulations are not applicable. In spite of the difficulties a good agreement between measurements and simulations was found when the flow speed was $50 \mathrm{~m} / \mathrm{s}$. In case of $26.8 \mathrm{~m} / \mathrm{s}$ flow speed it was found that the presence of the upper channel wall, not taken into account by the previous authors simulating this problem plays an important role - a hitherto unexplained peak in the measured spectrum appeared this way in the simulation.
\end{abstract}

\section{Keywords}

Vehicle door gap $\cdot$ cavity flow $\cdot C F D \cdot C A A \cdot$ self-sustained oscillation · competing oscillation mechanism · channel resonance

\section{Bence Farkas}

MTA-BME Water Management Research Group, Department of Hydrodynamic Systems, Faculty of Mechanical Engineering, Budapest University of Technology and Economics, Múegyetem rkp. 3, H-1111 Budapest, Hungary

\section{György Paál}

Department of Hydrodynamic Systems, Faculty of Mechanical Engineering, Budapest University of Technology and Economics, Múegyetem rkp. 3, H-1111 Budapest, Hungary

e-mail: paal@hds.bme.hu

\section{Introduction}

If the flow passes an open cavity a shear layer is formed at the mouth. This shear layer can lose its stability which often leads to a self-sustained oscillation. It is generally an unwanted phenomenon due to the generated noise, vibration and the increasing drag force [9]. The first studies were motivated by military aircraft problems [20, 26] with the main focus on high Mach number flows. In the meantime, many other technical applications have been discovered and many of them operate at low Mach number. For example, sunroofs and door gaps in automobiles, closed side branches in gas pipelines, flow of a river between two impermeable spur dykes or musical instruments. Early basic research was done mainly on rectangular cavities or sometimes on axisymmetric ones [9]. Later, application-specific, more complex geometries also became interesting. Rockwell and Naudascher [25] classified cavity tones as fluid-dynamic, fluid-resonant and fluid-elastic cavity oscillations. Our topic relates only to the first two categories. We speak about fluid-dynamic or Rossiter mode when an aerodynamic feedback process is responsible for the self-sustained oscillation [26]. The oscillating shear layer crosses the cavity mouth and impinges on the trailing edge of the cavity. The interaction of the shear layer with the trailing edge produces acoustic waves that excite the oscillations of the shear layer at the entrance of the cavity, thus closing the feedback loop. Different Rossiter modes can be formed depending on the number of simultaneously traveling vortices in the shear layer. Based on this theory, Rossiter constructed a semi-empirical formula to predict the arising frequencies

$$
f_{n}=\frac{U_{\infty}}{L} \cdot \frac{n-\alpha}{\frac{1}{\kappa}+M}, \quad n=1,2, \ldots
$$

where $U_{\infty}$ is the flow speed, $M$ is the Mach number, $L$ denotes the cavity length, $n$ stands for the order of the Rossiter mode, finally $\alpha$ and $\kappa$ are the two empirical constants: $\alpha$ represents a time delay between the vortex arrival at the trailing edge and the emission of the acoustic disturbance, while $\kappa$ denotes the ratio of disturbance convection speed in the shear layer and $U_{\infty}$. This is probably the most widely used formula for frequency estimation of cavity tones, but it is in accordance with experimental data 
rather for $M>0.4$ [27].

In the case of fluid-resonant oscillations the cavity acts as an acoustic resonator interacting with the broadband shear layer noise, selectively amplifying the eigenfrequencies of the cavity. When the frequency of a fluid-resonant oscillation is close to a Rossiter mode, the two phenomena can join resulting in a lock-on state [24]. Shallow cavities, (i.e. $L / D>1$ where $D$ is its depth) may produce longitudinal waves (waves traveling between the leading edge and trailing edge walls), while deep $(L / D<1)$ cavities can produce transversal waves (waves traveling between the cavity floor and the mouth). It was found in East's experimental work on deep rectangular cavities [4] that for $M<0.18$ the first two Rossiter-modes are likely to lockon with the fundamental depth mode of the cavity resonance. The frequency of the fundamental depth-mode can be estimated from the inequality below

$$
0.15<f D / a<0.22
$$

(where $a$ denotes the speed of sound), in agreement with the former theoretical work of Plumblee et al. [20].

Beside the Rossiter and resonant frequencies, lower modulation frequencies were also found either experimentally [13] or numerically [7]. According to flow visualization experiments in [23] modulation frequencies are related to the alternating occurrence of different types of vortex-corner interactions: complete clipping, partial clipping, partial escape, complete escape. Typology is based on the proportion of the vortex entering the cavity after impingement. Since the entering structures are convected in the large recirculation eddy back to the shear layer, they influence the flow paths of the vortices in the shear layer, thereby closing the feedback loop [7, 15]. The recirculation zone is also responsible for the formation of $3 \mathrm{D}$ flow structures. The simultaneous presence of the curved streamlines and the boundary layer near the cavity wall awakes centrifugal instability [3], which often brings about longitudinal (Taylor-Görtler) vortices [8]. The distance between these subsequent longitudinal vortices are usually the same or the half of the wavelength of the primary (shear-layer) waves [22]. For further information on cavity tone, Gloerfelt's book [10] can be a good starting point.

There are two main strategies in Computational Aeroacoustics (CAA) to compute the noise generated by the flow: Direct Noise Computation (DNC) or hybrid methods. In the case of the DNC the transient compressible Navier-Stokes equations (optionally coupled with a turbulence model) are solved with a high precision. Since sound is in fact time-dependent pressure fluctuation, it can be obtained from the computed time-dependent pressure field. Hybrid methods, however, compute only the flow field in the first run. From these results, source terms are computed that form the right hand side of the inhomogeneous wave equation. Most acoustic methods are based on Lighthill's acoustic analogy [17, 18] and Green's function formalism. A widely used extension of this theory was invented by Ffowcs Williams and Hawkings [30]. With that the presence of moving walls also can be managed. This extension was also achieved with another integral formulation (Kirchhoff-Helmholtz) by Farassat and Myers [5]. The advantage of using hybrid methods is that the most appropriate domain size, mesh density and equation set can be used in each run which reduces the computational cost significantly. If the analyzed phenomenon allows to perform the flow simulation in the incompressible mode, then the lack of pressure waves will spare the use of non-reflective boundaries and the large computational domain that makes the simulation less resource-intensive.

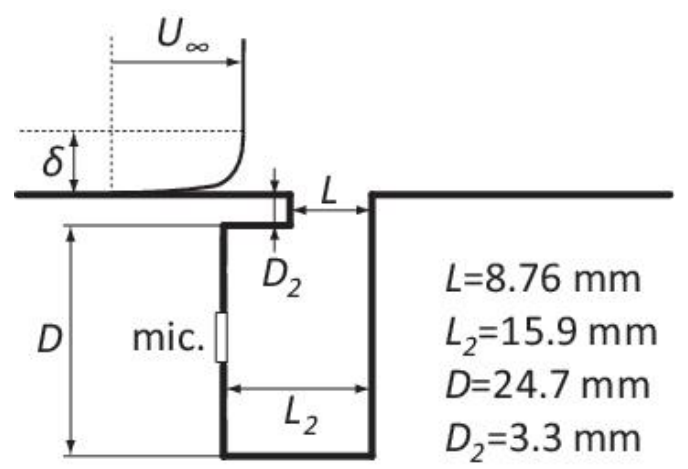

Fig. 1. Cavity configuration used in the experiments $[12$

\subsection{About the examined geometry and flow parameters.}

In this paper, our interest focuses on a deep cavity with a lip, which is actually a simplified model of an automobile door gap. Since this geometry was used on the Third Computational Aeroacoustics (CAA) Workshop on Benchmark Problems (Category 6), some papers on numerical efforts and the experimental results of Henderson are also available for validation [12]. The geometry parameters of the cavity are shown in Fig. 1 1 Originally the problem was defined for workshop participants with boundary layer thickness $(\delta)$ of $16 \mathrm{~mm}$ and $22 \mathrm{~mm}$ for the flow speeds $\left(U_{\infty}\right)$ of $26.8 \mathrm{~m} / \mathrm{s}$ and $50.9 \mathrm{~m} / \mathrm{s}$ respectively. Due to technical difficulties, however, the measurements were performed later with thinner boundary layers. Information on that is not entirely clear but probably $\delta=2 \mathrm{~mm}$ and $12 \mathrm{~mm}$ were obtained which were referenced as the 'thin' and the 'thick' cases in her paper. The flow speed was also changed to $50 \mathrm{~m} / \mathrm{s}$ from $50.9 \mathrm{~m} / \mathrm{s}$. The location of pressure signal capture was the center of the left wall of the cavity using two $1 / 4$ " condenser microphones and a dynamic signal analyzer [12].

Ashcroft et al. [2] used the CFL3D code for their compressible two-dimensional simulations with Wilcox's $k-\omega$ turbulence model. The flow-induced noise was computed with DNC. The flow parameters were $U_{0}=50.9 \mathrm{~m} / \mathrm{s}$ and $\delta=22 \mathrm{~mm}$. They used a multiblock mesh with $68.7 \mathrm{~K}$ cells and simple boundary conditions, thus only short time simulations were possible to avoid interference by wave reflections from the boundaries. This simulation produced a hydrodynamic shear layer oscillation with $5.5 \%$ higher frequency than that of Henderson (this comparison takes the different speeds into consideration and makes a linear correction). Moon et al. [19] applied the splitting method 
of Hardin and Pope [11]. For the first run the incompressible Navier-Stokes equation was solved (without a turbulence model). In the case of the $26.8 \mathrm{~m} / \mathrm{s}$ flow speed the (laminar) boundary layer thickness was $15 \mathrm{~mm}$, while in case of $50.8 \mathrm{~m} / \mathrm{s}$ flow speed a boundary layer thickness of $19 \mathrm{~mm}$ was used. The mesh for the first run consisted of $11.2 \mathrm{~K}$ triangular elements. In case of lower speed the shear layer operates in the first mode with $4 \%$ higher frequency than the experimental result, but in the higher speed case the frequency over-prediction is $12 \%$ compared to the dominant peak of Henderson's spectrum. Kurbatskii and Tam [16] applied DNS simulations with a very thin laminar boundary layer. The mesh was constructed from sub-domains. Moving away from the cavity the successive sub-domains always had twice as large cells than those in the previous one. A Dispersion-Relation-Preserving scheme was used which was able to deal with different cell sizes. It was also able to apply different timesteps in different sub-domains.

Our goal in this paper is to apply a commercial solver in combination with a commercial mesh generator to this benchmark problem, using our extensive experience in simulation of cavity flows. We aim at testing various meshes, various turbulence models, the limits and possibilities of 2D models vs 3D models and the non-reflecting boundary condition of the code since we are aware that only compressible simulations are feasible in this geometry.

\section{Numerical settings}

Our simulations were performed using the ANSYS CFX 14.0 commercial software and the meshes were constructed using ANSYS ICEM. Mainly compressible flow simulations were performed with non-reflecting boundary conditions, and the obtained pressure spectra were compared with experimental data. No separate acoustic simulation was performed.

\subsection{Computational domains and boundary conditions}

First 2D domains were used with different eddy-viscosity and Reynolds-stress turbulence models. A 2D domain for CFX consists of a single layer of 3D cells since the CFX solver technically always deals with $3 \mathrm{D}$ cells. After the $2 \mathrm{D}$ simulations, pseudo $3 D$ domains were constructed. The span-wise dimension of a pseudo 3D domain was much smaller than the cavity dimension, it was about $0.1 \mathrm{~mm}$ and it was resolved with 6-20 layers. This was necessitated by the DES (Detached Eddy Simulation) model which is not supposed to be applied in the case of a 2D mesh [32]. The aim of the pseudo 3D DES simulations were to form an intermediate step between 2D Reynolds-Stress and real 3D DES simulations. This may help distinguish between changes coming from the new turbulence model and changes coming from the new geometry. Finally, (real) 3D simulations were performed with both DES and Reynolds-stress turbulence models. The span-wise length of the cavity - used in Henderson's measurement - was $15 \mathrm{~cm}$. Modeling the whole flow domain with an appropriately resolved mesh was far beyond of our computational capacities (both in terms of memory and CPU). Ahuja and Mendoza [1] have studied the effect of span-wise length $(W)$ on the flow for rectangular cavities and they found that when $W$ exceeds the cavity length it has no further effect on the emitted sound of the flow. This finding had to be utilized very carefully in the case of a cavity with a lip, thus $W$ was chosen to be $15.9 \mathrm{~mm}$ which is the total length of the lip and $L$ and denoted with $L_{2}$ in Fig. 1 . Even so, serious compromises were necessary in order to limit computational costs.

Fig. 2 shows the computational domains for different approaches. The direction of the flow is always from left to right. The smallest area around the cavity was used for 2D incompressible simulations. For the bottom of the domain - including also the walls of the cavity - no slip wall boundary condition was prescribed. At the left boundary velocity inlet was used. The profile of the boundary layer was exported from an auxiliary stationary simulation with Shear Stress Transport (SST) turbulence model, since the analytical approximation with $1 / 7$ power law does not provide smooth and accurate data where the boundary layer and the uniform region connect. This auxiliary simulation had a 2 meter long domain and uniform velocity profile at the inlet, thus it was capable providing boundary layer profile data for a wide range of thicknesses. The desired thickness was always related to the mouth of the cavity, so the prescribed profile of the inlet had to be appropriately thinner. For the top and right boundary a pressure condition was prescribed without specifying the direction of the flow. (More precisely we used the following settings: Boundary type: Opening, Mass and momentum option: Entrainment, Pressure Option: Opening pressure see [32] for further details.) The other three domains in Fig. 2

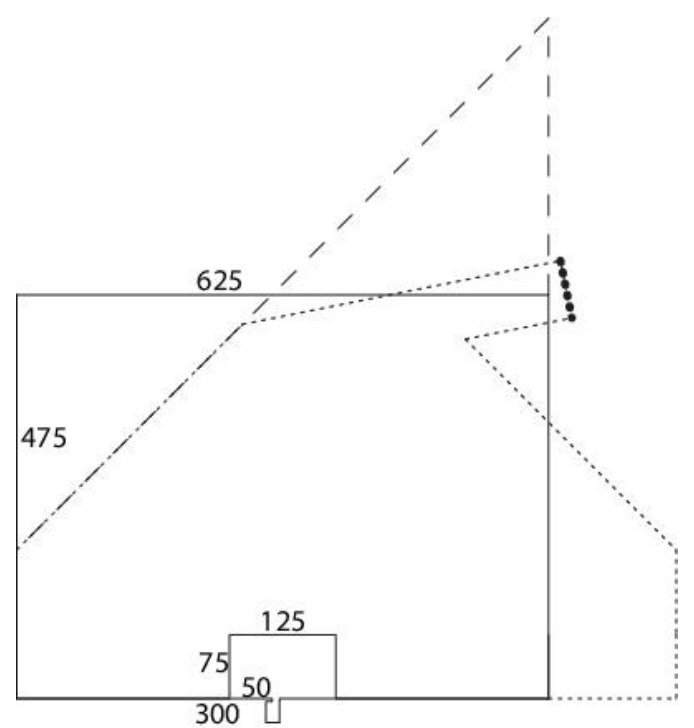

Fig. 2. Computational domains for the different approaches. (Distances are in millimeter.) Smallest area (solid border) for incompressible simulations, big rectangular area for initial compressible simulations. The area bounded with the dashed line was used when the 'opening pressure' boundary condition at the top had to be eliminated. Finally the area bounded with the dotted line was used when the downstream 'opening pressure' boundary condition had to be eliminated additionally. 
were stages of an evolutionary process relating to compressible simulations. In these cases enforcing the stable operation of the non-reflecting boundaries was the largest challenge which was not fully achieved. At the time of performing the simulations, the development of the built-in non-reflecting feature of ANSYS CFX was still in progress, version 14.0 contained it as a hidden beta function. According to a validation report [31] the implementation of the time-dependent non-reflecting boundaries was based on the characteristic analysis [21,28]. As a first attempt the big rectangular 2D domain was used (Fig. 2). The boundaries of this domain are more than one wavelength away from the cavity for any arising frequencies. The upstream (velocity inlet) non-reflecting boundary was stable, but the opening pressure boundaries especially at the top produced high frequency numerical instabilities which destroyed the simulations on the long term. Our reaction to this problem was to change the domain as depicted with the dashed line in the figure and substitute the top pressure boundary with a velocity inlet. These changes solved the numerical instability problems for 2D runs, but in case of 3D with the Detached Eddy Simulation turbulence model, fatal numerical instabilities developed at the downstream boundary (see Fig. 3(a)). A number of variations were tested both for the downstream boundary condition (different kinds of opening and outlet conditions) and for the mesh (improved mesh with excellent aspect ratio and uniform cell size near the downstream boundary) but none of them was effective in tackling the instability problem. Thus we were forced to prescribe mass flow or velocity at the downstream boundary instead of pressure. This necessitated another modification of the domain which is depicted with dotted line in Fig. 2 A short section remained pressure outlet in order to avoid global mass imbalance accumulation but this section was hidden from direct impact of waves. This part is depicted with larger dots in the figure. Two kinds of attempts were made with this domain in order get rid of these instabilities. First mass outlet was prescribed instead of pressure, but in this case the velocity profile was not stationary: it produced large scale oscillations at the outlet. The other attempt was to prescribe the velocity profile at the downstream boundary. The profile was acquired from an auxiliary simulation which was performed on the same domain but with an incompressible fluid model. Surprisingly the prescribed velocity profile was largely violated by the simulation in the long term as depicted in Fig. 3 (b). It seams that while the solver ensures non-reflectivity the calculated velocity profile deviates from the prescribed one. Fortunately approximately 100 shear layer oscillations occur before losing stability, so that many useful conclusions can be deduced from these simulations.

\subsection{Mesh density}

Mesh dependence study had to be performed several times during this work because of the wide variety of the used approaches. In some former works of the authors, thorough mesh studies were made on rectangular cavities [6]. The initial struc- ture of the mesh (relative densities of different regions, aspect ratio of the cells, etc.) was based on this experience. First, meshes were purely block structured, but later the structured regions with different densities were connected with hybrid blocks (called swept blocks in ICEM). The usage of this meshing strategy allowed the avoidance of interfaces and cells with bad aspect ratio in spite of the large differences in cell size for different regions. The initial mesh study was related to $2 \mathrm{D}$ incompressible flow. The main mesh parameters are collected in Table 1 It was found that both oscillation frequency and amplitude converged if the mesh size was larger than $26.4 \mathrm{~K}$ cells. On coarser meshes both parameters decreased. Thus, for later mesh dependence studies the mesh densities in the second part of the table served as a guideline. Generally the density - defined by the mesh with $26.4 \mathrm{~K}$ cells - proved to be sufficient also in other cases. As for the far-field mesh the rule of thumb was used that prescribes 20 cells along one wavelength. Considering the relevant frequencies, a $4.5 \mathrm{~mm}$ cell size was chosen.

Tab. 1. Mesh properties in case of incompressible simulations. The last two columns show the horizontal and the vertical cell sizes in the shear layer

\begin{tabular}{cccc}
\hline $\begin{array}{c}\text { number } \\
\text { of cells }\end{array}$ & $\begin{array}{c}\text { cells along } \\
\text { shear layer }\end{array}$ & $\begin{array}{c}\text { size } \leftrightarrow \\
{[\mathrm{mm}]}\end{array}$ & $\begin{array}{c}\text { size } \uparrow \\
{[\mathrm{mm}]}\end{array}$ \\
\hline $8.4 \mathrm{~K}$ & 40 & 0.33 & 0.045 \\
\hline $10.6 \mathrm{~K}$ & 47 & 0.22 & 0.045 \\
\hline $14.5 \mathrm{~K}$ & 55 & 0.18 & 0.038 \\
\hline $19.5 \mathrm{~K}$ & 64 & 0.15 & 0.033 \\
\hline \hline $26.4 \mathrm{~K}$ & 74 & 0.13 & 0.028 \\
\hline $36 \mathrm{~K}$ & 86 & 0.11 & 0.024 \\
\hline $49 \mathrm{~K}$ & 100 & 0.09 & 0.02 \\
\hline
\end{tabular}

Fig. 4(a) shows the final 2D mesh in the close neighborhood of the cavity. It can be seen that the boundary layer is resolved before the cavity. A region after the cavity, which is largely affected by vortex shedding is also very well resolved. Further downstream the cell size continuously grows up to the regular cell size of the far-field mesh. The main reason for the elimination of the boundary layer mesh far downstream of the cavity, is to provide cells with a good aspect ratio at the downstream boundary of the flow region. This is important because of the applied non-reflecting boundary condition in the case of compressible simulations. Fig. 4(b) shows the mesh at the mouth of the cavity. It can be observed that the horizontal resolution of the spacious part of the cavity is coarser than that of the bottleneck region which was achieved with a thin swept block. In case of $3 \mathrm{D}$ meshes this swept blocks were essential in order to reduce the mesh size. Fig. 5 shows the places where the coarsening in different directions was performed in 3D. A fine span-wise resolution was restricted only in the very close neighbourhood of the cavity for reasons of computational economy. The Detached Eddy Simulation (DES) turbulence model [32] was an exception. This model performed Large Eddy Simulation in the separated flow and classical RANS elsewhere. DES required about $0.1 \mathrm{~mm}$ cell size to treat the whole volume of the cavity 


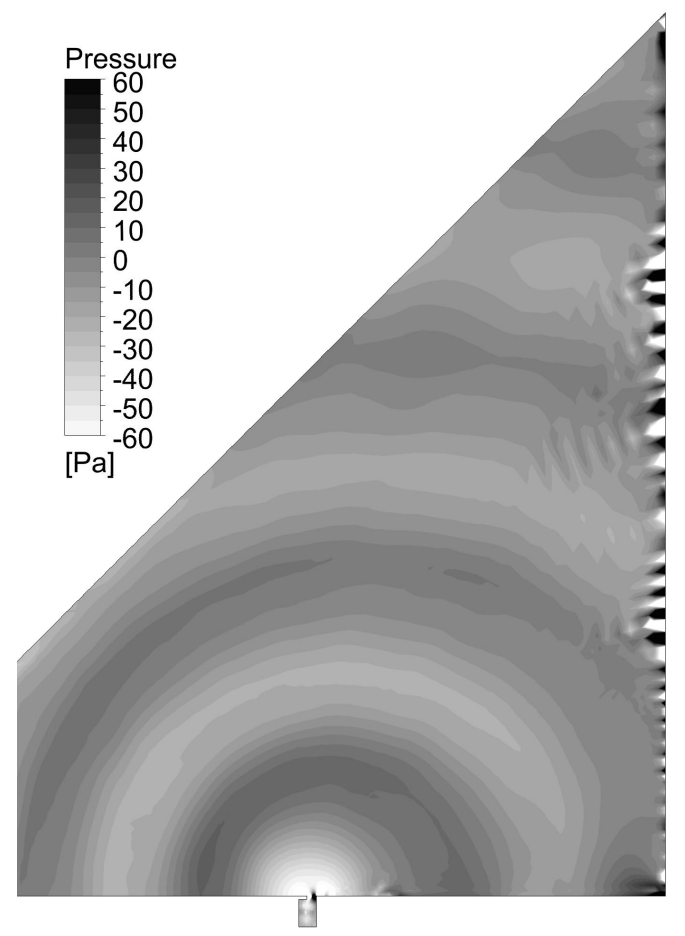

(a)

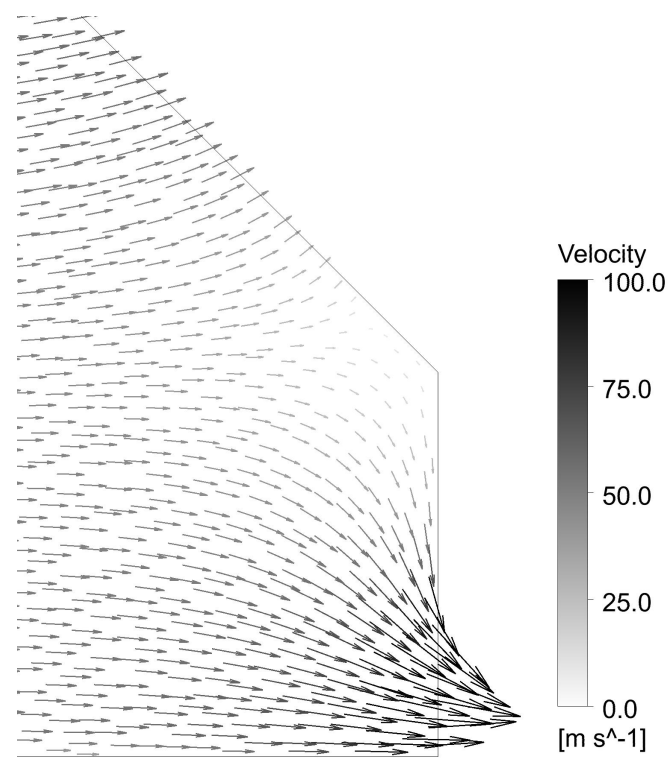

(b)

Fig. 3. Arising numerical instabilities of the $3 \mathrm{D}$ DES runs

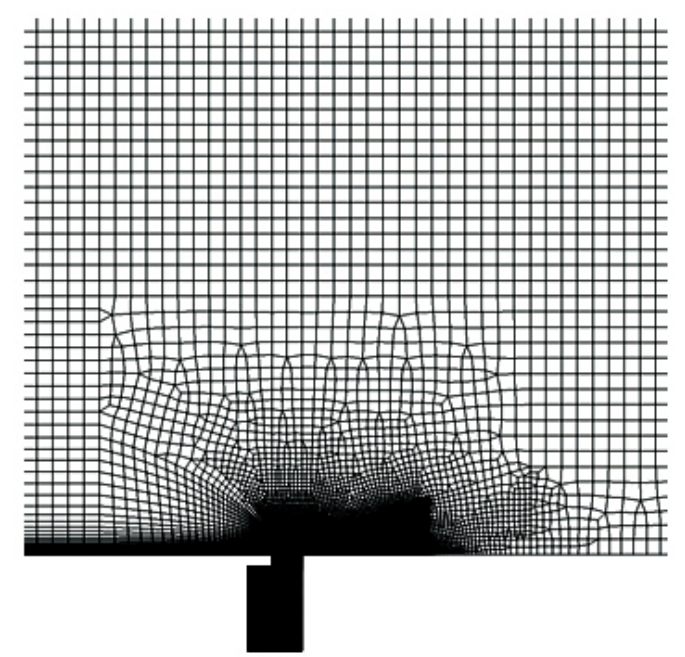

(a) 2D mesh in the close vicinity of the cavity

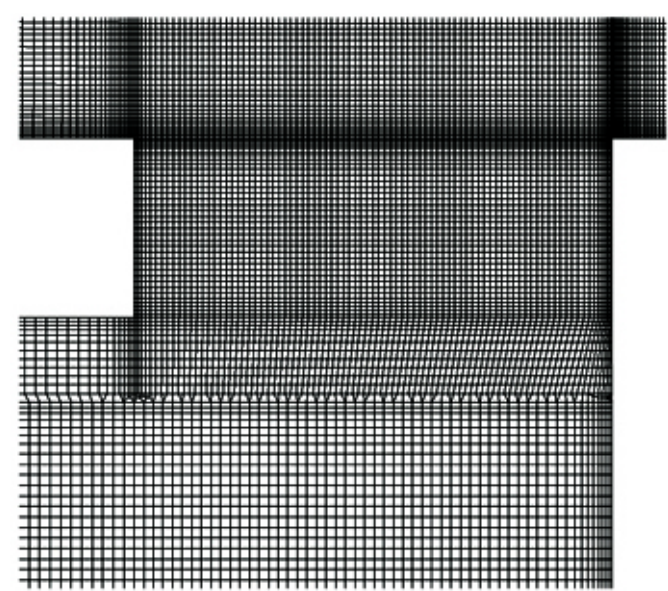

(b) 2D mesh at the mouth of the cavity

Fig. 4. 


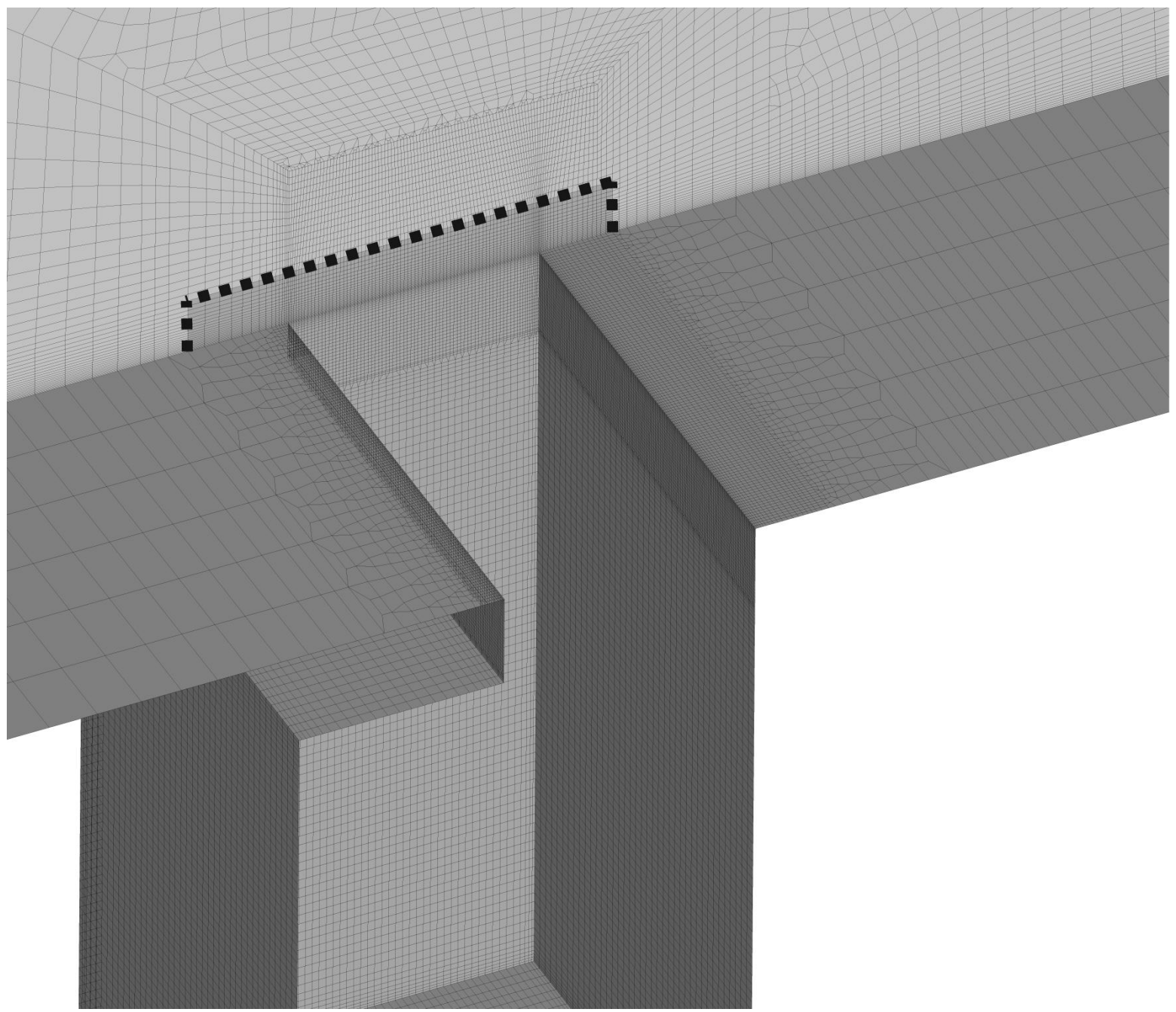

Fig. 5. 3D mesh

as a detached region and to use the LES method there instead of RANS. Thus for these runs the coarsening of the spacious part of the cavity was applied to a lesser extent.

\subsection{Time step}

For the few incompressible simulations - with the meshes listed in Table 1- the appropriate timestep ensured that the RMS Courant number (based on the local flow velocity) was below 1 . To achieve this, the timestep had to be set between 1.5 and $9 \mu$ s depending on the mesh. In other cases the matter of timestep was revisited several times. For compressible simulations the timestep of $1 \mu$ s was appropriate in general. Again DES simulations were most demanding, since there was a qualitative change in the flow when the timestep was reduced to $0.5 \mu \mathrm{s}$ from $1 \mu \mathrm{s}$.

\section{Results and discussion}

\subsection{Effect of viscosity}

No temperature value was published associated with the reference measurements, although it affects the Reynolds number through the viscosity. A short parametric study was performed on the temperature to detect its possible impact on the flow. Changing the air temperature between 0 and $50{ }^{\circ} \mathrm{C}$ caused no significant change in the shear layer oscillation frequency in ac- cordance with a similar experimental result [1]. Frequency differences were within $2 \%$ and no linear tendency was observed, while the Reynolds number $\left(R e_{L}=U_{\infty} L / v\right)$ varied between 29006 and 32932. The amplitude of the pressure oscillations was also independent of the Reynolds number. The finding about frequencies cautions about using the Reynolds number as a characteristic parameter of the flow in these cases. In the same experimental study it was also found that higher air temperature leads to higher noise levels contrary to our simulational results.

\subsection{Effect of boundary layer thickness}

The majority of the workshop participants used thinner boundary layers than Henderson did during the reference measurement for the 'thick' case (probably $12 \mathrm{~mm}$ ). Usually it was necessary to counterbalance some numerical over-dissipation and force shear layer oscillation since thick incoming boundary layer is one of the best antidote of oscillation [1]. In agreement with similar studies [16] we have found, that thinning the boundary layer increases not only the amplitude but also the frequency of the shear layer oscillation (see Fig. 6). That explains why frequency over-prediction was so frequent among the results of workshop participants. Our results, however, suffer from the opposite error in case of $\delta \geq 10 \mathrm{~mm}$. Coincidence with the 'thick' 
reference result (denoted with dashed line in Fig. 6) would be possible with a boundary layer thickness of 8-9 $\mathrm{mm}$.

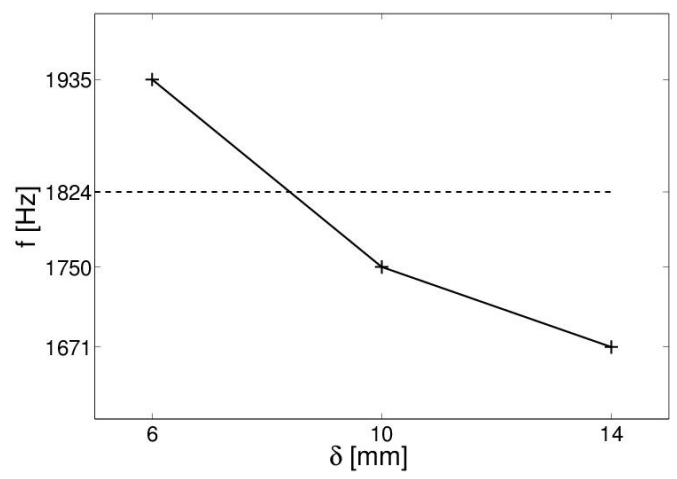

Fig. 6. Effect of boundary layer thickness on the fundamental shear layer oscillation frequency at $U_{\infty}=50 \mathrm{~m} / \mathrm{s}$. The dashed line represents the experimental result of [12]

\subsection{Inadequacy of incompressible flow simulation for this configuration}

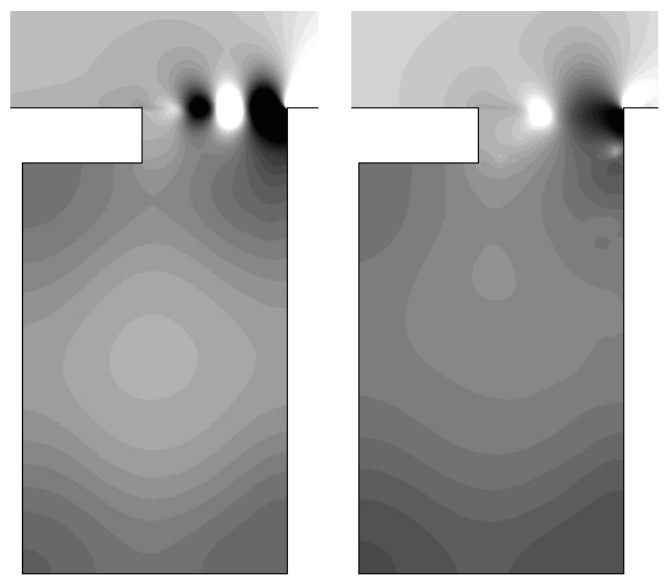

Fig. 7. Pressure contours in case of $U_{\infty}=50 \mathrm{~m} / \mathrm{s}$. Left: incompressible, right: compressible

In case of $U_{\infty}=50 \mathrm{~m} / \mathrm{s}$ the result of an incompressible simulation was qualitatively different from the result of a compressible one (all other parameters unchanged). The compressible model produced $1^{\text {st }}$ shear layer mode (as expected from Rossiter's formula and Henderson's measurements) while the incompressible simulation resulted in a $2^{\text {nd }}$ mode oscillation as evidenced by Fig. 7. The wrong result of the incompressible simulation was persistent while perturbing $U_{0}$ and $\delta$ values by about $\pm 20 \%$ and trying different turbulence models. This circumstance warns that applying the hybrid CAA method with an incompressible fluid-dynamic simulation can easily produce erroneous results in cases where acoustical feedback on the flow is significant. Wang et al. [29] also experienced this problem by the same geometry and flow parameters.

\subsection{Turbulence model comparison}

A wide variety of built-in turbulence models were tested assuming compressible fluid. The applied eddy viscosity models $(k-\omega, \mathrm{SST})$ did not produce unsteady behavior in the low speed
$(26.8 \mathrm{~m} / \mathrm{s})$ and thick boundary layer $(12 \mathrm{~mm})$ case where oscillations do take place according to Henderson's measurements. In case of higher speeds $\left(U_{\infty}=50 \mathrm{~m} / \mathrm{s}\right)$ the unsteady behavior appeared but the under-prediction of the arising frequencies was more significant than that for the Reynolds-stress models. The Scale Adaptive Simulation (SAS) suffered from the same issues in 3D. The BSL Reynolds-stress model had more but still not enough willingness to go unsteady. The most appropriate models for the 2D simulation were the SSG Reynolds-stress and the $\omega$ Reynolds-stress models with which no spurious steady behaviour was detected.

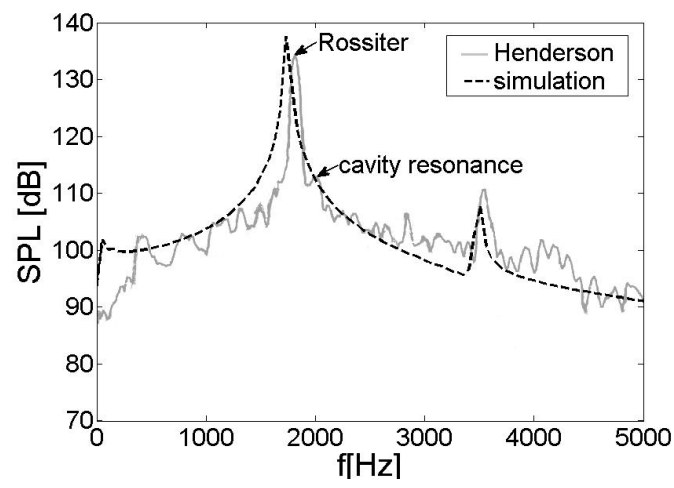

Fig. 8. Spectra in case of $U_{\infty}=50 \mathrm{~m} / \mathrm{s}$. For the $2 \mathrm{D}$ simulation $\mathrm{SSG}$ turbulence model was used with $\delta=10 \mathrm{~mm}$.

In case of $U_{\infty}=50 \mathrm{~m} / \mathrm{s}$ the amount of under-prediction for the spectral peaks was $3.5-5 \%$ while the amplitude over-prediction of the dominant peak ( $1^{\text {st }}$ Rossiter mode) was $4 \mathrm{~dB}$ as shown in Fig. 8. Only the fluid-dynamic oscillation appeared (with its $1^{s t}$ harmonic) in the spectrum and there was no sign of any acoustic modes contrary to Henderson's spectrum, which contains a small peak at $2016 \mathrm{~Hz}$. Considering also other results - which will be discussed in the next paragraph - it can be stated that simulating competitive mechanisms was generally unsuccessful: one of the mechanisms totally suppressed the others. In spite of this, Fig. 8 shows a very good agreement with Henderson's experiment because in case of $U_{\infty}=50 \mathrm{~m} / \mathrm{s}$ the missing cavity resonance mode is very weak compared to the Rossiter mode. In case of $U_{\infty}=26.8 \mathrm{~m} / \mathrm{s}$ the power of these two mechanisms are more balanced, thus the coincidence of the simulational results were not so good there (Fig. 10, Table 2). The authors are aware of two successful attempts to simulate competitive mechanisms for this benchmark problem [14,16], however those ones were performed with laminar boundary layer and with much higher order numerical scheme.

\subsection{D flow structures}

To obtain the missing qualitative properties of the $2 \mathrm{D}$ results 3D simulations with $L_{2} / W=1$ ( $L_{2}$ was defined in Fig. 1) and periodic boundary conditions were tried. Fig. 9 shows very clearly the formation of longitudinal vortices in the recirculation zone. Shade reveals that fluid particles rotate in these longitudinal vortex tubes and shows also the direction of the rotation. These results imply that the Taylor-Görtler mechanism is responsible 


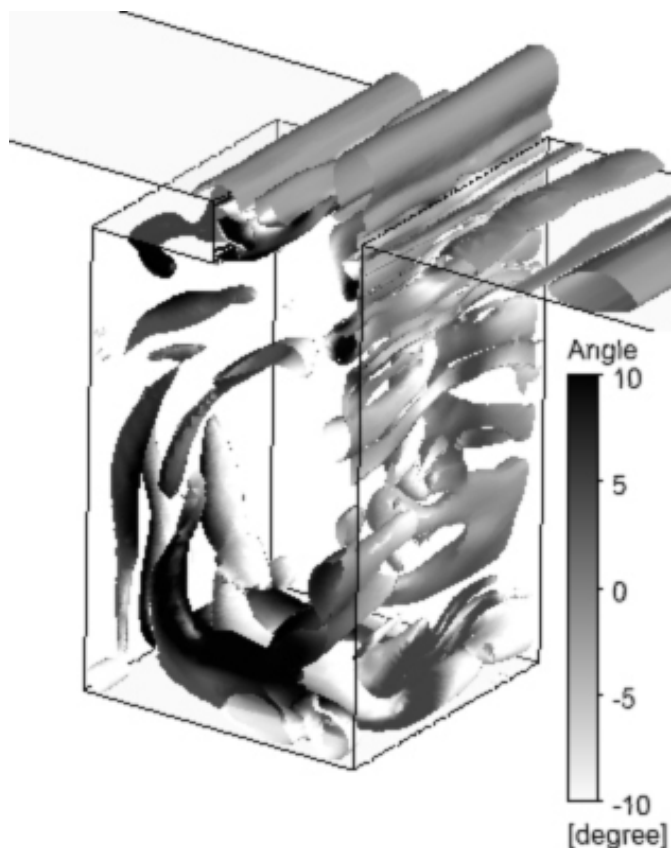

Fig. 9. $Q=0.032$ isosurface in case of $U_{\infty}=50 \mathrm{~m} / \mathrm{s}$ applying the DES turbulence model and periodic boundary condition. Shade represents the angle between the flow direction and the symmetry plane.

for the formation of these longitudinal vortices. This is in accordance with the literature [3, 8]. Immediately after impingement on the downstream edge the wavelength of the initial longitudinal vorticity was approximately half of the shear layer oscillation wavelength (both outside and inside the cavity) in agreement with the experimental result of Rockwell and Knisely [22]. As expected, the DES model had an advantage in illustrating the fine 3D structures compared to the Reynolds-stress models.

Beside the significant 3D flow structures it was also found that the lip of the cavity saved the shear layer from the direct impact of disturbances advected by the big recirculation zone. The recirculation zone was not able to interact with the shear layer as it is usual in rectangular cavities [7],15], thus the shear layer retained its two-dimensionality. Since the primary sound source is located at the downstream impingement edge of the cavity (where the flow remained essentially 2D), no additional frequency arose from the 3D nature of the flow. We note that our spectrum is not in perfect qualitative agreement with Henderson's measurements therefore one should consider these result with some caution. The frequency prediction of the 3D DES simulation was slightly worse that that of the 2D SSG Reynoldsstress simulation.

\subsection{Effect of the test section wall of the wind tunnel}

In case of $U_{\infty}=26.8 \mathrm{~m} / \mathrm{s}$ and 'thick' boundary there were two specialties in Henderson's spectrum. First, the reported $1^{\text {st }}$ mode Rossiter oscillation at $1168 \mathrm{~Hz}$ and the transversal cavity waves at $1984 \mathrm{~Hz}$ were very close in amplitude $(99$ and $101 \mathrm{~dB}$ respectively) as shown in Fig. 10 . Second, another peak appeared at $1890 \mathrm{~Hz}$ in Henderson's spectrum with the highest amplitude $(103 \mathrm{~dB})$. This peak is said to have unknown origin in the lit- erature.

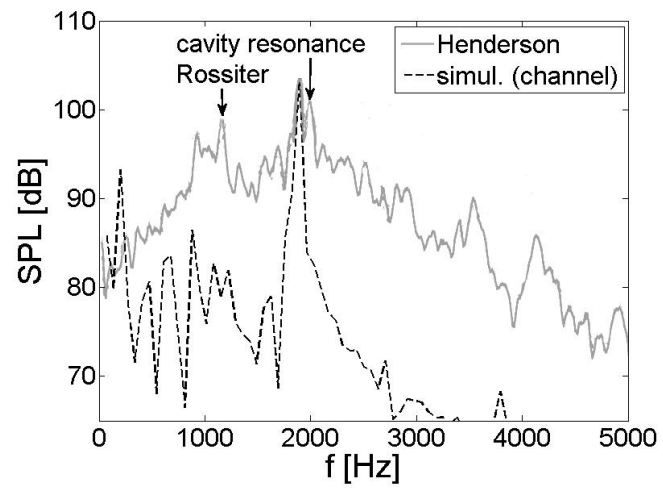

Fig. 10. Spectra in case of $U_{\infty}=26.8 \mathrm{~m} / \mathrm{s}$. For the $2 \mathrm{D}$ simulation BSL turbulence model was used with $\delta=12 \mathrm{~mm}$.

Is has already been mentioned that simulating competitive mechanisms was unsuccessful. A lot of different simulations were carried out but the result was always a single peaked spectrum (except for the higher harmonics) with a shear layer in the $2^{\text {nd }}$ Rossiter mode. We assume that false lock-on states had developed despite the big difference between the original frequencies of the competitive mechanisms. Some of these erroneous results are collected in Table 2 in order to demonstrate the wide variety of the resulting frequencies.

The enforcement of the $1^{\text {st }}$ Rossiter mode was closest in case of the 3D DES simulation where one of the two feedback cycles of the $2^{\text {nd }}$ Rossiter mode became significantly weaker than the other one but its total elimination was not achieved. This only resulted in a strong subharmonic component of the $2^{\text {nd }}$ Rossiter frequency in the spectrum and a pressure signal similar to the complete clipping - partial escape pattern [23]. Despite the shortcomings of the above-mentioned simulations an important finding was achieved while searching for the causes of this deficiency.

Henderson performed measurements in a wind tunnel with a test section of 48 " $\times 18$ " $\times 18$ ", thus the distance of the top wall of the test section was less than 3 wavelengths with respect to the dominant frequency both for high and low speed simulations. Therefore reflections from the top wall could have an influence on the flow. Surprisingly - as far as the authors aware - nobody has studied this effect until now.

In a 2D study the simulational domain was set to 48 " $\mathrm{x} 18$ " and the boundary condition at the top was set to a simple no slip wall. (The upstream and downstream boundaries remained nonreflecting as before.) Fig. 11 demonstrates the reflections from the top wall. The solid lines (representing wave fronts) were painted manually on the grayscale pressure field.

The most valuable conclusion about modeling the channel (the test section of the wind tunnel) arose when the BSL Reynolds-stress turbulence model was used with $\delta=12 \mathrm{~mm}$ and $U_{\infty}=26.8 \mathrm{~m} / \mathrm{s}$. As described before, a simulation with this turbulence model did not produce organized shear layer oscillation at low speed and 'thick' boundary layer. The energy of the 
Tab. 2. Dominant frequencies in case of $U_{\infty}=26.8 \mathrm{~m} / \mathrm{s}$ and 'thick' boundary layer for different modeling approaches and different numerical settings. The main experimental frequencies (depicted also in Fig. 10) were: $1890 \mathrm{~Hz}(103$ $\mathrm{dB}), 1984 \mathrm{~Hz}(101 \mathrm{~dB})$ and $1168 \mathrm{~Hz}(99 \mathrm{~dB})$.

\begin{tabular}{ccccc}
\hline geometry & $\begin{array}{c}\delta \\
{[\mathrm{mm}]}\end{array}$ & $\begin{array}{c}\text { turbulence } \\
\text { model }\end{array}$ & $\begin{array}{c}\text { oscillation } \\
\text { type }\end{array}$ & $\begin{array}{c}\mathrm{f} \\
{[\mathrm{Hz}]}\end{array}$ \\
\hline 2D & 10 & $\omega$ Reynolds-stress & $2^{\text {nd }}$ Rossiter & 1311 \\
\hline pseudo 3D & 10 & DES & $2^{\text {nd }}$ Rossiter & 1486 \\
\hline pseudo 3D & 14 & $\begin{array}{c}\text { SSG } \\
\text { Reynolds-stress }\end{array}$ & $2^{\text {nd }}$ Rossiter & 1502 \\
\hline 3D & 14 & DES & $2^{\text {nd }}$ Rossiter & 1538 \\
\hline pseudo 3D & 10 & $\omega$ Reynolds-stress & $2^{\text {nd }}$ Rossiter & 1659 \\
\hline 2D channel & 10 & $\begin{array}{c}\text { SSG } \\
\text { Reynolds-stress }\end{array}$ & $2^{\text {nd }}$ Rossiter & 1817 \\
\hline 2D channel & 12 & $\begin{array}{c}\text { BSL } \\
\text { Reynolds-stress }\end{array}$ & channel resonance & 1890 \\
\hline
\end{tabular}

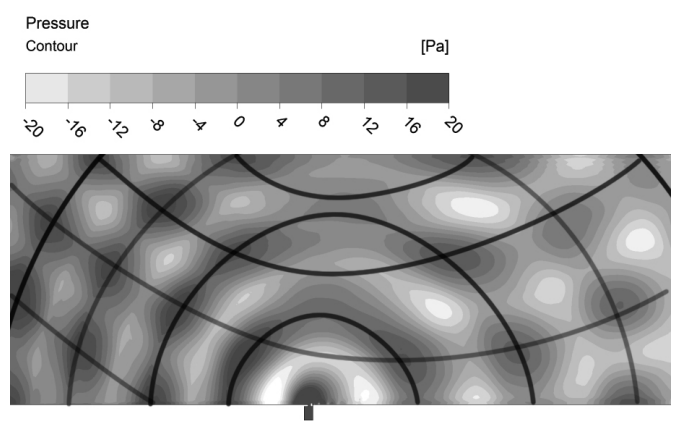

Fig. 11. Reflection of the pressure waves from the wall of the test section

main flow still nourished a periodic pressure fluctuation which was not present in the channel-free simulations. Fig. 10 demonstrates that both the frequency and amplitude of this new oscillation perfectly coincides with the highest peak in Henderson's spectrum (whose origin has been unexplained origin until now). These findings imply that this peak is associated with an acoustic resonance of the cavity-channel system. There are examples in the literature [24] that the shear layer oscillation and the cavity resonance are simultaneously present, merging into a common lock-on frequency. In our case, however, Henderson's measurements showed that it does not happen, the separate mechanisms preserve separate frequencies. Therefore the penultimate row of Table 2 shows a false 'merged' frequency which is de-tuned by a significant amount compared to the 'pure' Rossiter frequency of the first row. (The results by the $\omega$ Reynolds-stress and the SSG Reynolds-stress models hardly differ.)

We think that it is unlikely that the acoustic waves shown in Fig. 11 have an unphysical origin since it completely agrees with physical intuition that waves are reflected from the ceiling and the fact that its frequency agrees numerically with the measured frequency further confirms its physical nature. The question might arise why only this turbulence model reproduced this frequency peak and not the others. To this question we do not have complete answer but we can conjecture the following. All other turbulence models reproduced more or less the Rossiter frequency but none of them the cavity resonance or the channel resonance. In case of the BSL Reynolds stress model the shear layer oscillation might have been damped more than in the other cases and this allowed the formation of the channel mode since experience showed that simultaneous competing modes could not be simulated. We have not tried 3D simulations in this case.

\section{Conclusions}

Computational simulations were performed on a simplified automobile door gap both in 2D and 3D with ANSYS CFX using different turbulence models and the built-in non-reflective boundary conditions. These boundary conditions operated well at the velocity inlet but showed instabilities in the long term in case of opening and outlet conditions. The examined geometry and flow parameters were defined for the Third Computational Aeroacoustics (CAA) Workshop on Benchmark Problems, thus extensive literature is available on this special issue. The arising frequencies were found to be independent of the temperature and thus of the viscosity which warns about the usability of the Reynolds number as a single charasteristic parameter of the flow. Thinning the boundary layer, however, increases both oscillation frequency and amplitude. Comparing incompressible and compressible simulations at $U_{\infty}=50 \mathrm{~m} / \mathrm{s}$ it turned out that incompressible simulation was operating (incorrectly) in the $2^{\text {nd }}$ Rossiter mode while compressible simulations produced the $1^{\text {st }}$ mode. This points out that a hybrid CAA method with an incompressible fluid-dynamical simulation can easily produce erroneous result in case of the current benchmark problem since omitting acoustic feedback can change the flow qualitatively. For 2D simulations Reynolds-stress turbulence models were superior to eddy-viscosity models; best results were achieved with the SSG Reynolds-stress and with the $\omega$ Reynolds-stress turbulence models. For 3D simulations both Reynolds-stress models and Detached Eddy Simulation (DES) produced 3D flow structures assuming to be the result of the Taylor-Görtler instabilities. The DES model had an advantage in illustrating the fine 3D structures, however in the frequency prediction it was worse than the Reynolds-stress models. The 3D flow structures were not able to interact with the shear layer because of the lip of the cavity, thus the flow in the shear layer region remained essen- 
tially 2D. Simulating competitive driving mechanisms (Rossiter and acoustic modes) simultaneously was unsuccessful in general; one mechanism suppressed all the others or erroneous lockon states evolved. This caused only small differences from Henderson's experimental results in case of $U_{\infty}=50 \mathrm{~m} / \mathrm{s}$ but it was a serious drawback in case of $U_{\infty}=26.8 \mathrm{~m} / \mathrm{s}$ where the competitive mechanisms play a more important role. Yet an important advancement was achieved in case of $U_{\infty}=26.8 \mathrm{~m} / \mathrm{s}$ in understanding the origin of the dominant frequency peak which was hitherto unknown. Including the test section of the wind tunnel it turned out, that this peak is associated with the acoustic resonance of the cavity-tunnel system.

\section{References}

1 Ahuja KK, Mendoza J, Effects of cavity dimensions, boundary layer, and temperature on cavity noise with emphasis on benchmark data to validate computational aeroacoustic codes, NASA, 1995.

2 Ashcroft GB, Takeda K, Zhang X, Computations of self-induced oscillatory flow in an automobile door cavity, In: Third Computational Aeroacoustics(CAA) Workshop on Benchmark Problems, Vol. 1, 2000.

3 Brès GA, Colonius T, Three-dimensional instabilities in compressible flow over open cavities, Journal of Fluid Mechanics, 599(1), (2008), 309-339, DOI $10.1017 /$ S0022112007009925

4 East LF, Aerodynamically induced resonance in rectangular cavities, Journal of Sound and Vibration, 3(3), (1966), 277-287, DOI 10.1016/0022460X(66)90096-4

5 Farassat F, Myers MK, Extension of Kirchhoff's formula to radiation from moving surfaces, Journal of Sound and Vibration, 123(3), (1988), 451-460, DOI $10.1016 / \mathrm{S} 0022-460 \mathrm{X}(88) 80162-7$

6 Farkas B, Paál G, Computational investigation on the oscillation frequencies of the shear layer over an open cavity, In: Vad $\mathbf{J}$ (ed.), Conference on Modelling Fluid Flow CMFF'09, Department of Fluid Mechanics, Budapest University of Technology and Economics; Budapest, Hungary, 09/09/200912/09/2009, pp. 674-681.

7 Farkas B, Paál G, Szabó KG, Descriptive analysis of a mode transition of the flow over an open cavity, Physics of Fluids, 24(2), (2012), 24, DOI $10.1063 / 1.3687242$

8 Faure TM, Adrianos P, Lusseyran F, Pastur L, Visualizations of the flow inside an open cavity at medium range Reynolds numbers, Experiments in Fluids, 42(2), (2007), 169-184, DOI 10.1007/s00348-006-0188-8

9 Gharib M, Roshko A, The effect of flow oscillations on cavity drag, Journal of Fluid Mechanics, 177, (1987), 501-530, DOI 10.1017/S002211208700106X

10 Gloerfelt X, Cavity noise, In: Anthoine J, Christophe $\mathbf{J}$ (eds.), Aerodynamic noise from wall-bounded flows, Vol. 1, von Karman Institute for Fluid Dynamics, 2009, http://sin-web.paris.ensam.fr/ squelettes/ref_biblio/Gloerfelt_VKI_2009a.pdf

11 Hardin JC, Pope DS, Sound generation by flow over a two-dimensional cavity, AIAA Journal, 33(3), (1995), 407-412, DOI 10.2514/3.12592

12 Henderson B, Automobile Noise Involving Feedback- Sound Generation by Low Speed Cavity Flows, In: Third Computational Aeroacoustics(CAA) Workshop on Benchmark Problems, Vol. 1, 2000.

13 Knisely C, Rockwell D, Self-sustained low-frequency components in an impinging shear layer, Journal of Fluid Mechanics, 116, (1982), 157-186, DOI 10.1017/S002211208200041X

14 Koh SR, Cho Y, Moon YJ, Aeroacoustic computation of cavity flow in selfsustained oscillations, KSME International Journal, 17(4), (2003), 590-598, DOI $10.1007 / \mathrm{BF} 02984460$

15 Kuo C-H, Huang S-H, Influence of flow path modification on oscillation of cavity shear layer, Experiments in Fluids, 31(2), (2001), 162-178, DOI 10.1007/s003480100270

16 Kurbatskii K, Tam C, Direct numerical simulation of automobile cavity tones, In: Third Computational Aeroacoustics(CAA) Workshop on Benchmark Problems, 2000.

17 Lighthill J, On sound generated aerodynamically. II. Turbulence as a source of sound, Proceedings of the Royal Society of London. Series A. Mathematical and Physical Sciences, 222(1148), (1954), 1-32, DOI 10.1098/rspa.1954.0049

18 Lighthill J, On sound generated aerodynamically. I. General theory, Proceedings of the Royal Society of London. Series A. Mathematical and Physical Sciences, 211(1107), (1952), 564-587, DOI 10.1098/rspa.1952.0060

19 Moon YJ, Koh SR, Cho Y, Chung JM, Aeroacoustic computations of the unsteady flows over a rectangular cavity with a lip, In: Third Computational Aeroacoustics(CAA) Workshop on Benchmark Problems, Vol. 1, 2000.

20 Plumblee HE, Gibson JS, Lassiter LW, A theoretical and experimental investigation of the acoustic response of cavities in an aerodynamic flow, DTIC Document, 1962.

21 Poinsot TJ, Lele SK, Boundary conditions for direct simulations of compressible viscous flows, Journal of Computational Physics, 101(1), (1992), 104-129, DOI 10.1016/0021-9991(92)90046-2

22 Rockwell D, Knisely C, Observations of the three-dimensional nature of unstable flow past a cavity, Physics of Fluids, 23(3), (1980), 425-431, DOI 10.1063/1.863009

23 Rockwell D, Knisely C, Vortex-edge interaction: Mechanisms for generating low frequency components, Physics of Fluids, 23, (1980), 239-240, DOI $10.1063 / 1.862962$

24 Rockwell D, Lin JC, Oshkai P, Reiss M, Pollack M, Shallow cavity flow tone experiments: onset of locked-on states, Journal of Fluids and Structures, 17(3), (2003), 381-414, DOI 10.1016/S0889-9746(02)00141-X

25 Rockwell D, Naudascher E, Self-sustained oscillations of impinging free shear layers, Annual Review of Fluid Mechanics, 11(1), (1979), 67-94, DOI 10.1146/annurev.fl.11.010179.000435

26 Rossiter JE, Wind-tunnel experiments on the ow over rectangular cavities at subsonic and transonic speeds, Her Majesty's Stationery Office, London (1966), Aeronautical Research Council Reports and Memoranda, 1964, Report No.:3438.

27 Tam CKW, Block PJW, On the tones and pressure oscillations induced by flow over rectangular cavities, Journal of Fluid Mechanics, 89(02), (1978), 373-399, DOI $10.1017 /$ S0022112078002657

28 Thompson KW, Time-dependent boundary conditions for hyperbolic systems, II, Journal of Computational Physics, 89(2), (1990), 439-461, DOI 10.1016/0021-9991(90)90152-Q

29 Wang ZK, Djambazov G, Lai CH, Pericleous K, Numerical simulation of flow-induced cavity noise in self-sustained oscillations, Computing and Visualization in Science, 10(3), (2007), 123-134, DOI 10.1007/s00791-0060039-4

30 Williams JEF, Hawkings DL, Sound generation by turbulence and surfaces in arbitrary motion, Philosophical Transactions of the Royal Society of London. Series A, Mathematical and Physical Sciences, 264(1151), (1969), 321342, DOI 10.1098/rsta.1969.0031

31 Zwart $\mathbf{P}$, Abshoff $\mathbf{J}$, Non-reflective Boundary Conditions in CFX-5, Ansys Canada Ltd., 2005, Report No.:CFX-VAL01/07042/10/2005.

32 ANSYS CFX-Solver Modeling Guide, Release 14.0, ANSYS, Inc.; Southpointe, 275 Technology Drive, Canonsburg, PA 15317, 2011. 